\title{
Mental health diagnoses in adults with phenylketonuria: a retrospective systematic audit in a large UK single centre
}

\author{
George Altman ${ }^{1,2^{*}+}$,, Kamran Hussain ${ }^{1 \dagger}$, Diane Green ${ }^{2}$, Boyd J. G. Strauss ${ }^{1,3}$ and Gisela Wilcox ${ }^{1,2}$
}

\begin{abstract}
Background: Recently published European Society for Phenylketonuria (ESPKU) guidelines have recommended a lifelong diet with phenylalanine (Phe) control $\leq 600 \mu \mathrm{mol} / \mathrm{L}$ for phenylketonuria (PKU) patients. This study aimed to identify whether PKU adult patients are at a higher risk of mental health diagnoses if their 2-year average Phe level is higher than the ESPKU European guidelines. Published studies identified by a literature review showed that related studies have been published in American and European PKU study populations but not in the United Kingdom (UK) study populations. Previous studies also involved a smaller number of participants due to this being a rare disease.

Results: We undertook a retrospective audit at a single large PKU centre in the UK. 244 adult PKU patients at the centre were included, 220 of which had a recorded Phe level. Approximately $75 \%$ of the patients in this study did not meet the ESPKU European guidelines for Phe control. A systematic search of the electronic patient record was undertaken looking for mental health diagnoses. Compared to two-year average Phe levels $\leq 600 \mu \mathrm{mol} / \mathrm{L}$, PKU adult patients with two-year average Phe levels $>600 \mu \mathrm{mol} / \mathrm{L}$ were more likely to have diagnoses of low mood, depression, anxiety, or mood swings, but only low mood reached statistical significance $(p<0.05)$.
\end{abstract}

Conclusions: PKU patients with two-year average Phenylalanine levels greater than ESPKU guidelines may be at greater risk of mental health diagnoses and symptoms. Many of these adult PKU patients will be lost to follow-up, and therefore may be receiving treatment for mental health conditions in the community. Multicentre UK studies and international collaborations are required to overcome low participant numbers in the study of this rare disease.

Keywords: Phenylketonuria, Phenylalanine, Neuropsychiatric, Mental health, Depression, Anxiety, Mood swings, Low mood, Audit, ESPKU guidelines

\section{Background}

Phenylketonuria (PKU) is an autosomal recessive inherited metabolic disorder. Patients with the condition have an absent or dysfunctional phenylalanine hydroxylase (PAH) [1]. The deficiency in PAH results in high phenylalanine (Phe) levels in the blood. Excess phenylalanine

\footnotetext{
*Correspondence: George.altman@mft.nhs.uk

${ }^{\dagger} J$ oint first authors: George Altman and Kamran Hussain.

${ }^{1}$ Faculty of Biology, Medicine and Health, University of Manchester, Manchester, UK

Full list of author information is available at the end of the article
}

is toxic to the developing brain and competes with other large neutral amino acids e.g. tryptophan for crossing the blood-brain barrier. Together with deficient tyrosine, this causes marked neurotransmitter derangement, with deficiencies of dopamine, noradrenaline, and serotonin [2]. In the UK, an estimated 1 in 10,000 newborn babies will have PKU [3]. Currently, the only treatment available for all PKU patients in the UK is a lifelong phe restricted diet. This diet was first conceptualised by Professor Isaac Woolf using casein hydrolysate treated with active carbon to produce a dietary supplement low in Phe [4]. Woolf worked with Dr Horst Bickel to treat the first patient with 
this diet at Birmingham Children's Hospital [5]. PKU patient outcomes were revolutionised by this therapeutic diet. Extreme natural protein restriction, supplemented with micronutrient-fortified phenylalanine-free amino acid-based supplements to meet nutritional requirements is instituted neonatally [2]. Close blood-spot monitoring of Phe levels, maintained throughout development, has enabled the attainment of near-potential IQ [6]. However, adherence to such dietary stringency is difficult for many, and alternative therapies e.g. tetrahydrobiopterin (BH4) or Kuvan ${ }^{\circledR}$, co-factor for PAH, are limited to those with residual enzyme activity, demonstrated responsiveness, and access [6-9]. Furthermore, it is estimated that half of all patients in the UK with inherited metabolic disease have been lost to follow-up; this means there may be a significant number of patients no longer on the diet, who are receiving care only within a general practice setting [10]. It is essential to determine the impact of high Phe levels on mental health diagnoses, as there may be a considerable unidentified burden of mental health disease in this population.

In 2017, the ESPKU guidelines were published, which advised targeting Phe $\leq 600 \mu \mathrm{mol} / \mathrm{L}$ lifelong, with a lower target during pregnancy and preconception [2]. The evidence level was graded at D; therefore further studies are required to improve the strength of evidence for this guideline. In view of controversy amongst key opinion leaders in the relationship between concurrent Phe control and mental health, and the relative lack of evidence in adults. We sought to investigate, in our large unselected patient cohort, if a relationship could be observed between prevailing phenylalanine control and the prevalence of mental health symptoms [2]. We used a method of keyword searches to extract recorded mental health symptoms as recorded by clinicians in the electronic patient record, a form of real world evidence.

This study asked the following research question: are adult patients with PKU at higher risk of mental health diagnoses if their average Phe level is higher than the ESPKU European Guidelines of $\leq 600 \mu \mathrm{mol} / \mathrm{L}$ ? The null hypothesis is that there is no significant difference in the prevalence of mental health diagnoses between patients with a 2-year average Phe level above and below the ESPKU Guidelines of $\leq 600 \mu \mathrm{mol} / \mathrm{L}$. A single-centre retrospective clinical audit at the Mark Holland Metabolic Unit, Salford Royal NHS Foundation Trust, Manchester, United Kingdom was undertaken.

\section{Literature review}

A literature review was undertaken to identify previous methodologies used to answer this research question. Studies were filtered to include adult patients with a diagnosis of PKU. Relevant outcomes sought were the prevalence of mental health diagnoses in this population (low mood, anxiety, depression, and mood swings). Published studies in the last 30 years, investigating the relationship between Phe levels and mental health diagnoses were included in the literature review.

The search strategy involved a database search using a scoping review. Boolean logic was used to combine keywords: Depression, Anxiety, Mood, PKU, Phenylketonuria, Phenylalanine. The search utilised three databases: Pubmed, Google Scholar, and the University of Manchester library. The final method for identifying additional papers was citation chaining.

Six papers met the search criteria [11-16]. In terms of validity, there was a wide range of study designs. In terms of reliability, two studies found a significant relationship between Phe levels and neuropsychiatric symptoms [11, 12]; one study found a non-statistically significant relationship and three studies found no relationship [13-16]. In terms of applicability, none of the studies included patients from the UK, which limits the application of these results to a UK PKU population (Fig. 1).

\section{Method of audit}

A systematic registered audit was undertaken using the clinical database maintained by the Mark Holland Metabolic Unit at the Salford Royal NHS Foundation Trust. The database contained 244 adult patients with PKU; 24 patients had no recorded Phe levels in the last two years and were excluded from the audit. Seventeen patients had a pregnancy during the previous two years; an additional separate analysis of pregnant PKU patients was included since pregnant patients have different targets regarding Phe control $(<360 \mu \mathrm{mol} / \mathrm{L})$.

The 2-year average Phe level was used as the measure of Phe control. Calculation of the average used all measurements recorded on the electronic patient record, or clinic letters, during the previous two years. All outpatient clinical letters in the electronic medical record were combined as a single document and searched in the preceding 2 years for the terms:

"Depression", "Anxiety", "Low Mood" and "Mood swings". This included both longstanding diagnoses recorded in the problem list as well as emerging diagnoses or symptoms recorded in the consultation by the clinician. If a clinician used the terms in a list of diagnoses or a problems list at any point in the 2 years prior to our data collection, this was recorded as a positive result. Patient age, sex, and pregnancy status were also recorded. Odds ratios (with $95 \%$ confidence intervals), were calculated to establish the association between Phe level $>600 \mu \mathrm{mol} / \mathrm{L}$ and mental health diagnoses. The data was independently double-checked for accuracy by both first authors. 


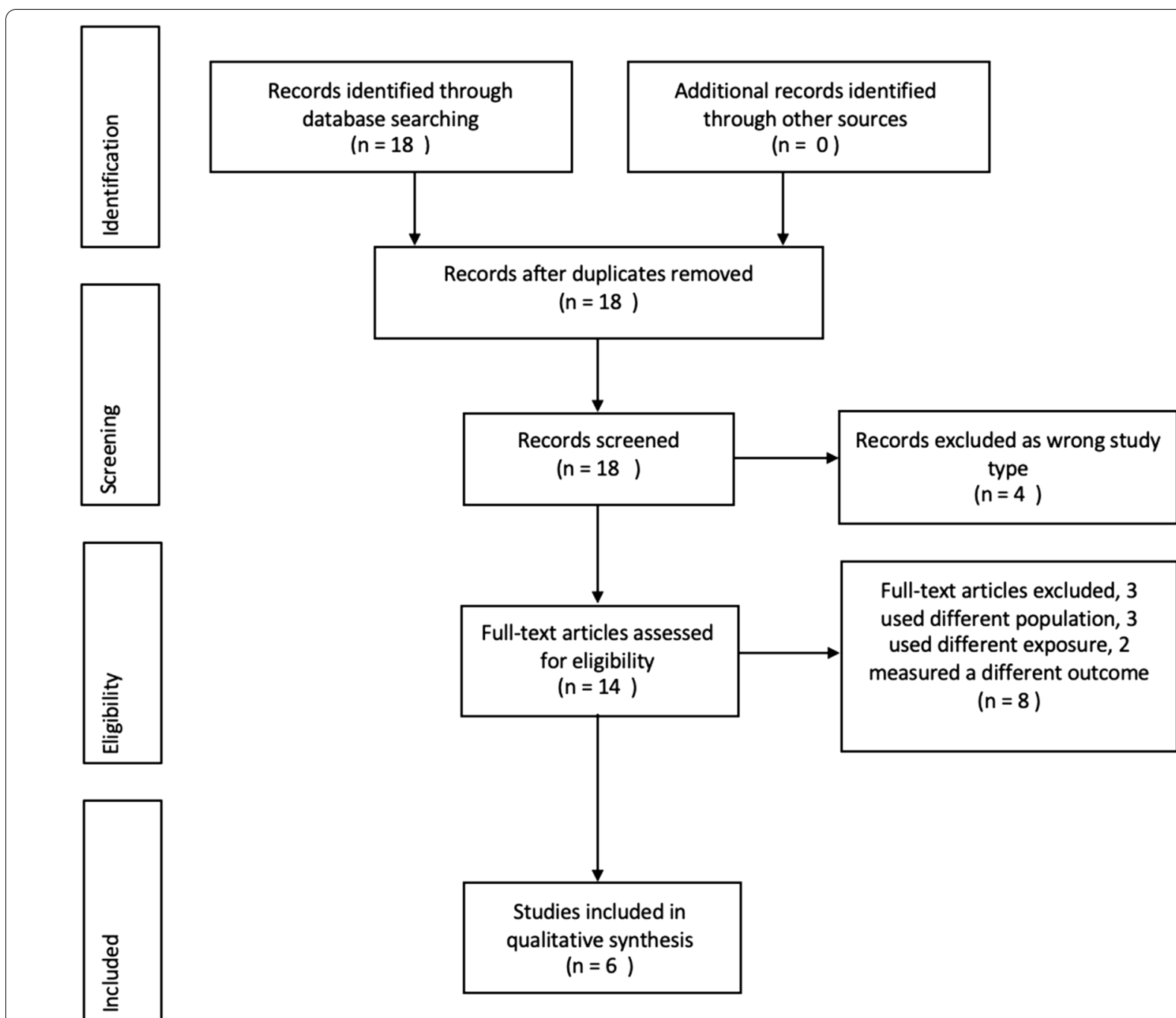

Fig. 1 Adapted from PRISMA Flow chart for literature search—from: Moher D, Liberati A, Tetzlaff J, Altman DG, The PRISMA Group (2009). Preferred reporting items for systematic reviews and meta-analyses: The PRISMA statement. PLoS Med 6(7): e1000097. 10.1371/journal.pmed1000097

\section{Results}

Our initial exploratory analysis demonstrated several notable trends throughout the extent of the dataset. The mean age was 35.9 years with a range of $18-71$ years. 27 patients were born before 1969 when national heelprick test screening started in the UK. Before this Phenistix nappy testing was used which missed an estimated $25-50 \%$ of cases. Therefore it is likely that some of these patients were delayed in starting treatment [17]. The data evidencing the precise extent of such a delay was not available, however, the mean prevalence of mental health symptoms in patients born before heelprick testing was $33.3 \%$, compared with $16.3 \%$ in those born after the advent of heelprick testing. The mean number of Phe readings was 7.1 over two years, with a range of $1-70$. The mean Phe level was $944.6 \mu \mathrm{mol} / \mathrm{L}$ (standard deviation $443.46 \mu \mathrm{mol} / \mathrm{L}$ ) and a range of 162 $2003 \mu \mathrm{mol} / \mathrm{L}$. Forty-nine of 202 patients had a 2-year average Phe $\leq 600 \mu \mathrm{mol} / \mathrm{L}$ i.e. $24.6 \%$ of non-pregnant patients were adhering to ESPKU European Guidelines [2]. 9/17 pregnant patients had a two-year average Phe level $<360 \mu \mathrm{mol} / \mathrm{L}$ during pregnancy and preconception. Therefore $52.9 \%$ of pregnant patients were adhering to ESPKU guidelines.

\section{Mental health symptoms}

Odds ratios (with 95\% confidence intervals), were calculated to establish the association between Phe level $>600 \mu \mathrm{mol} / \mathrm{L}$ and mental health diagnoses. Overall, 31(14.1\%) of non-pregnant patients had an electronic 
Table 1 Patients with a depression diagnosis in each group of phenylalanine control

\begin{tabular}{lccc}
\hline $\begin{array}{l}\text { Depression } \\
\text { diagnosed }\end{array}$ & Phe $>\mathbf{6 0 0} \boldsymbol{\mu m o l} / \mathbf{L}$ & Phe $\leq \mathbf{6 0 0} \boldsymbol{\mu m o l} / \mathbf{L}$ & Total \\
\hline Yes & 26 & 4 & 30 \\
No & 128 & 45 & 173 \\
Total & 154 & 49 & 203 \\
\hline
\end{tabular}

Odds ratio: $2.9885 .95 \% \mathrm{Cl} 1.0001-8.9301$. z statistic: 1.960

Significance level $P=0.0500$

Table 2 Patients with a anxiety diagnosis in each group of phenylalanine control

\begin{tabular}{lccc}
\hline $\begin{array}{l}\text { Anxiety } \\
\text { diagnosed }\end{array}$ & Phe $>\mathbf{6 0 0} \boldsymbol{\mu m o l} / \mathbf{L}$ & Phe $\leq \mathbf{6 0 0} \boldsymbol{\mu m o l} / \mathbf{L}$ & Total \\
\hline Yes & 34 & 6 & 40 \\
No & 120 & 43 & 163 \\
Total & 154 & 49 & 203 \\
\hline
\end{tabular}

Odds ratio: 2.2358. 95\% Cl: 0.9352-5.3450. z statistic: 1.809

Significance level $P=0.0704$

Table 3 Patients with a low mood diagnosis in each group of phenylalanine control

\begin{tabular}{lccc}
\hline $\begin{array}{l}\text { Low mood } \\
\text { diagnosed }\end{array}$ & Phe $>\mathbf{6 0 0} \boldsymbol{\mu m o l} / \mathbf{L}$ & Phe $\leq \mathbf{6 0 0} \boldsymbol{\mu m o l} / \mathbf{L}$ & Total \\
\hline Yes & 27 & 4 & 31 \\
No & 127 & 45 & 172 \\
Total & 154 & 49 & 203 \\
\hline
\end{tabular}

Odds ratio: 3.1231. 95\% Cl: 1.0475-9.3114. z statistic: 2.043

Significance level $P=0.0410$

medical record diagnosis of depression. 42(19.1\%) patients had a diagnosis of anxiety. 32(14.5\%) patients had a diagnosis of low mood, and 22(10\%) patients had a diagnosis of mood swings.

\section{Odds ratios including pregnant women Depression}

See Table 1.

\section{Anxiety}

See Table 2 .

\section{Low mood}

See Table 3.

\section{Mood swings}

See Table 4.
Table 4 Patients with a mood swings diagnosis in each group of phenylalanine control

\begin{tabular}{lccc}
\hline $\begin{array}{l}\text { Mood swings } \\
\text { diagnosed }\end{array}$ & Phe $>\mathbf{6 0 0} \boldsymbol{\mu m o l} / \mathbf{L}$ & Phe $\leq \mathbf{6 0 0} \boldsymbol{\mu m o l} / \mathbf{L}$ & Total \\
\hline Yes & 19 & 2 & 21 \\
No & 135 & 47 & 182 \\
Total & 154 & 49 & 203 \\
\hline
\end{tabular}

Odds ratio: 4.3478. 95\% Cl: 0.9850-19.1924. z statistic: 1.940

Significance level $P=0.0524$

Table 5 Table showing odds ratios relative to the lowest quartile of Phe levels for each mental health diagnosis, 95\% confidence interval, and $P$ value

\begin{tabular}{llcl}
\hline Diagnosis/symptom & Odds ratio & $\mathbf{9 5 \%} \mathbf{C l}$ & Significance \\
\hline Depression & 2.9 & $1-8.9$ & $P=0.0500$ \\
Anxiety & 2.2 & $0.93-5.35$ & $P=0.0704$ \\
Low mood & 3.1 & $1.04-9.31$ & $P=0.0410$ \\
Mood swings & 4.3 & $0.98-19.1$ & $P=0.0524$ \\
\hline
\end{tabular}

\section{Diagnosis/symptom}

See Table 5 and Fig. 2.

\section{Discussion}

Possible mechanisms of development of mental health conditions in PKU

There are a number of ways in which phenylketonuria may impact neuropsychological functioning and mood. This includes both short-term and long-term effects, both biological and psychosocial.

Long-term developmental effects may result from the cumulative impact of elevated blood phenylalanine competitively disrupting the transport of other large neutral amino acids (LNAA) across the blood brain barrier (BBB) via the LAT1 transporter [2]. These LNAA include essential amino acids required for protein and myelin synthesis as well as tyrosine which can be considered conditionally essential in PKU. Tyrosine and tryptophan, required for dopamine and serotonin synthesis respectively, are reduced in their CNS uptake and furthermore, phenylalanine elevation inhibits the enzymatic activity required to synthesise those specific neurotransmitters. The various biological mechanisms of the neuropsychological impact of PKU are described in further depth and breadth in the comprehensive review by Ashe et al. [18].

Whilst early-treated patients may be significantly protected from the major impact of severe hyperphenylalaninaemia, the effects of elevated phenylalanine levels on neurotransmitter production persist, potentially 
Prevelance of mental health symptoms in each group of Phe control

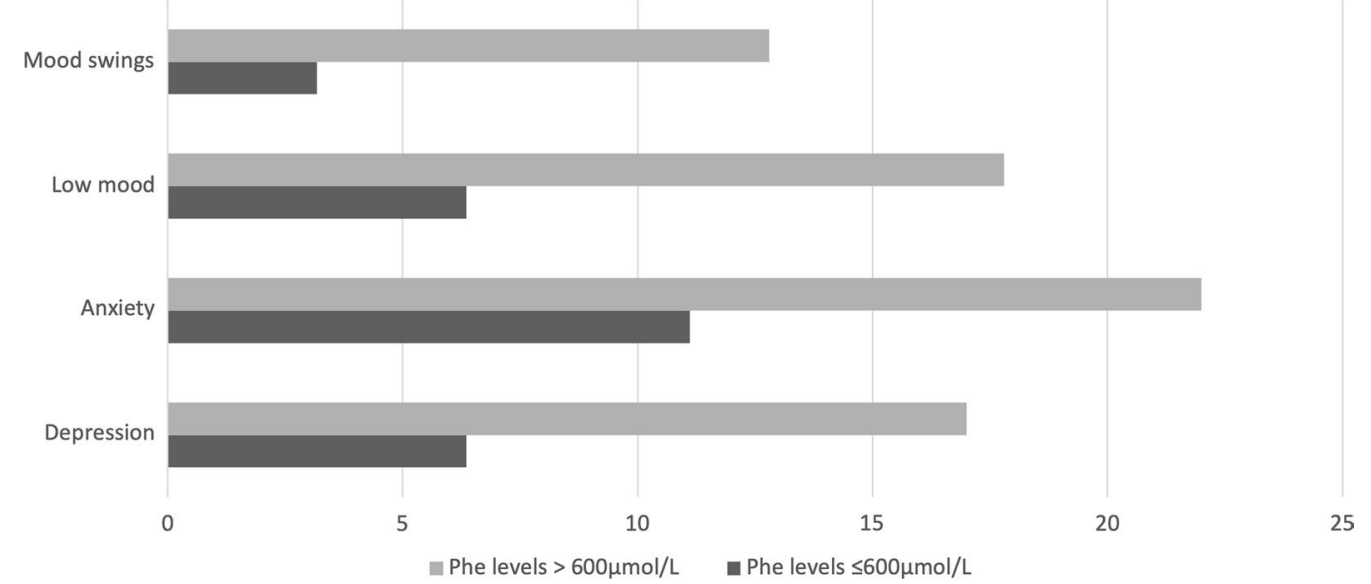

Fig. 2 Shows the percentage of patients with mood swings, low mood*, anxiety, or depression in patients with 2-year average Phe levels $>600 \mu \mathrm{mol} / \mathrm{L}$ and $\leq 600 \mu \mathrm{mol} / \mathrm{L} .{ }^{*} P<0.05$

impacting mood and executive function. These mechanisms may underlie the observations in our unselected PKU patient cohort as well as those reported in the literature [2]. Our findings of a possible relationship between contemporaneous phenylalanine control and clinically recorded mental health symptoms, reaching statistical significance with respect to low mood, might reflect short to medium term effects of hyperphenylalanimaemia but longitudinal studies in such patients might be needed to differentiate short-term versus long-term effects.

Independent of phenylalanine control, significant psychosocial factors are impacting on the mental health of our PKU patients. Some of these relate to the issues of having a rare condition, requiring an unusual and highly restrictive diet, and the intra-familial stress and other social stresses that follow [5]. Our study was not designed to capture these longer-term aspects of PKU.

The current first-line treatment for depression and anxiety is the use of selective serotonin reuptake inhibitors. Due to the reduced availability of serotonin, this first-line treatment may not be as effective in PKU patients, Ashe et al. identified no studies of antidepressant effectiveness in PKU patients in their review article [18].

\section{Limitations of the current literature}

There are several limitations of the currently available literature on the relationship between Phe levels and neuropsychiatric symptoms in PKU. First, there is an over-reliance on studies containing low numbers of participants. This is to be expected with a rare disease. In the future, multicentre studies should be used to overcome this limitation. Second, the literature review does not represent the UK PKU population, since, to our knowledge, no studies on the mental health of PKU patients have been completed in the UK. The age of participants is generally young, which does not represent the entire PKU population. Bilder et al. suggest a positive correlation between age and the presence and intensity of neuropsychiatric symptoms in PKU patients [13]. A young adult study population may risk underestimating the burden of neuropsychiatric symptoms in the whole PKU population. Third, the literature reviewed used a wide range of different questionnaires and screening tools which may not be directly comparable. Last, there is no standard measure of Phe levels with studies using a range of methods (current, 6-month average, 2-year average) which may obscure the relationship between Phe levels and neuropsychiatric symptoms. As the randomised control trial was the strongest form of evidence available for the current literature review, there is strong support for a link between Phe levels and neuropsychiatric symptoms [11]. However, further work needs to be done to consider the impact of a range of confounding factors (socioeconomic status, age, gender, and other biomarkers such as tyrosine and tryptophan levels).

\section{Results including pregnant patients}

All four mental health diagnoses have an odds ratio of $>1$, therefore exposure to two-year average Phe of $>600$ is associated with higher odds of each outcome (depression, anxiety, low mood, mood swings). The analysis of low mood has a 95\% confidence interval of 1.0475-9.3114, demonstrating a significant result. However, there is a low level of precision as the odds ratio range is large. Depression overlaps with 1 but has a significance level of 0.05 thus representing a strong association. With regard 
to anxiety and mood swings, the association of Phe $>600$ and increased prevalence of these conditions did not reach significance but shows a strong trend despite the limitations and exploratory nature of the study.

\section{Results excluding pregnant patients}

All four mental health diagnoses have an odds ratio $>1$, therefore exposure to 2 year average Phe of $>600$ is associated with higher odds of each outcome (depression, anxiety, low mood, mood swing). Despite this, the association between Phe and mental health symptoms did not reach significance in this group.

\section{Benefits of the study}

The study used audit data of all PKU patients in the clinic database and is thus a form of real-world evidence. Using real-world evidence reduces the likelihood of selection bias [19, 20]. However, patients may still have been selected in some form, as patients will be removed from the database if they miss two clinic appointments, this procedure may have 'selected' for more motivated patients who are more likely to engage with healthcare professionals. The evidence is drawn from a wider group of patients than previous studies in the literature review which focused on younger PKU patients aged 20-30. This study has a wider age range which may allow for better appreciation of changes in mental health diagnoses in older PKU patients but may still have missed those PKU patients whose diagnosis may have been missed before the introduction of Guthrie testing in the late 1960s. Ageing in treated PKU patients is currently an area with little evidence available as treated patients are only just reaching their fifth and sixth decade [21]. This study used a larger population of patients than previous studies, giving the study greater power to find relationships between the exposure and outcome. Finally, this study is the first using a UK PKU population, studying this population may identify specific trends in the UK PKU population.

\section{Limitations of the audit}

The comprehensiveness of the dataset with regard to the multitude of factors influencing Phe control prevents us from establishing causality. In addition, there may be an issue of reverse causality due to timelines; this could occur if a PKU patient with depression subsequently commenced a low Phe diet. The patient may still be recorded as having a diagnosis of depression but would now have a lower Phe level. However, Burgess et al. identified improvements in anxiety and depression ratings after the reintroduction of dietary control of PKU showing causality between reduced Phe levels and improving mental health symptoms suggesting reverse causality may be unlikely in this case [22]. However, the direction of the association between Phe level and mental health diagnoses remains uncertain in our patient group.

Several variables were not controlled for and may be confounding including age, gender, socioeconomic circumstances, tyrosine/tryptophan levels. The study also relies on the accuracy of the patient record. There are many sources of error in electronic patient records, including incorrect data entry and the introduction of incorrect diagnoses which are not later removed [23].

There may also be hidden time effects present; if there is seasonal variation in the prevalence of mental health symptoms, then the two-year sampling may not have equally sampled seasons with increased incidence of anxiety or depression [24].

Mental health conditions may be underdiagnosed; patients with borderline or subthreshold anxiety or depression may not be detected in the clinic. Therefore the results may underestimate the prevalence of anxiety and depression.

A final consideration is the impact of diet on mental health. Other chronic diseases which involve restricted diet eg diabetes, have an increased prevalence of mental health diagnoses [25]. The PKU diet is significantly more restricting, and patients may find it time-consuming and socially isolating [5]. Being on a Phe restricted diet may itself be a source of stress and anxiety [26]. This could increase the prevalence of anxiety in patients with low Phe and confound the result.

\section{Conclusion}

In this audit, $\cong 75 \%$ of adults Phe control did not meet the $\leq 600 \mu \mathrm{mol} / \mathrm{L}$ target outlines in the European Guidelines. The hypothesis put forward was that patients with PKU were at higher risk of mental health diagnoses if their average Phe levels were higher than the ESPKU guidelines of $\leq 600 \mu \mathrm{mol} / \mathrm{L}$. There is a statistically significant association between patients exposed to a twoyear average Phe $>600 \mu \mathrm{mol} / \mathrm{L}$ and a diagnosis of low mood. PKU patients with Phe $>600 \mu \mathrm{mol} / \mathrm{L}$ were more likely to have a diagnosis of depression, anxiety, or mood swings, but these trends were not statistically significant. However, the underdiagnosis of depression and anxiety in clinical settings may contribute to this outcome. Those whose Phe levels were greater than the guidelines appeared to have greater morbidity with respect to mental health symptoms, but larger studies including a prospective multicentre audit are needed to confirm this observation.

This audit represents the largest in terms of sample size to date, and, to our knowledge, the first to investigate mental health diagnoses in a UK PKU population. The implications for future research are that multicentre studies are required to improve the precision and power 
of the research. Future studies should aim to include consideration of confounding factors and use study designs that can avoid reverse causality such as a case-control study. In addition, the use of a more detailed dataset including details such as mean time to presentation from diagnosis, socioeconomic status, or region would be of greater utility in establishing causality, beyond the associations we have demonstrated. Expanding the assessment of mental health symptoms to include verified rating scales and diagnostic interviews would enable a greater level of precision when comparing these with the contemporaneously recorded Phe levels.

\section{Acknowledgements}

We thank Dr Matthew Sperrin for his statistical advice after the initial data collection. We thank the SSIEM 2018 conference for allowing us to present part of this research as a poster presentation.

\section{Authors' contributions}

GW conceived of the study, and participated in its design and coordination. $\mathrm{GA}, \mathrm{KH}, \mathrm{GW}, \mathrm{BS}$, and DG participated in the design of the study and performed the statistical analysis. GA and KH designed and conducted the data collection. GA, KH, GW, BS helped to draft the manuscript. All authors read and approved the final manuscript.

\section{Funding}

No funding to declare,

\section{Availability of data and materials}

Please contact author for data requests.

\section{Declarations}

\section{Ethics approval and consent to participate}

Audit registered with Salford Royal NHS Trust—Audit number 2017-171. Ethical approval not applicable as unidentifiable data collected during audit.

\section{Patient consent}

Not applicable.

\section{Competing interests}

Dr George Altman, Dr Kamran Hussain, Diane Green, Prof Boyd JG Strauss: none to declare. Dr Gisela Wilcox — has received travel grants from Genzyme, Biomarin, Alexion, Shire \& Amicus, speaker honoraria from Sanofi-Genzyme, Vitaflo, Biomarin, Shire/Takeda and Nutricia, research grants from the MPS society (UK), and advisory board membership with Biomarin \& Meta Healthcare, Medical Advisory Panel membership for the National Society for PKU (NSPKU) and consultancies for Dimension Therapeutics.

\section{Author details}

${ }^{1}$ Faculty of Biology, Medicine and Health, University of Manchester, Manchester, UK. ${ }^{2}$ The Mark Holland Adult Inherited Metabolic Disorders Unit, Ladywel Building, Salford Royal NHS Foundation Trust, Stott Lane, Salford M6 8HD, UK. ${ }^{3}$ Department of Medicine, School of Clinical Sciences, Faculty of Medicine, Nursing and Health Sciences, Monash University, Melbourne, Australia.

Received: 10 August 2021 Accepted: 28 November 2021

Published online: 20 December 2021

\section{References}

1. Williams RA, Mamotte CDS, Burnett JR. Phenylketonuria: an inborn error of phenylalanine metabolism. Clin Biochem Rev. 2008;29(1):31-41.

2. van Spronsen FJ, van Wegberg AMJ, Ahring K, Bélanger-Quintana A, Blau N, Bosch AM, et al. Key European guidelines for the diagnosis and management of patients with phenylketonuria. Lancet Diabetes Endocrinol. 2017. https://doi.org/10.1016/s2213-8587(16)30320-5.

3. Loeber JG, Gerard L. Neonatal screening in Europe; the situation in 2004 J Inherit Metab Dis. 2007. https://doi.org/10.1007/s10545-007-0644-5.

4. Alonso-Fernández J, Woolf LI. At the forefront of newborn screening and the diet to treat phenylketonuria-biography to mark his 100th birthday. Int J Neonatal Screen. 2020. https://doi.org/10.3390/ijns6030061.

5. Ford S, O'Driscoll M, MacDonald A. Living with phenylketonuria: lessons from the PKU community. Mol Genet Metab Rep. 2018. https://doi.org/ 10.1016/j.ymgmr.2018.10.002.

6. van Wegberg AMJ, MacDonald A, Ahring K, Bélanger-Quintana A, Blau N, Bosch AM, et al. The complete European guidelines on phenylketonuria: diagnosis and treatment. Orphanet J Rare Dis. 2017. https://doi.org/10. 1186/s13023-017-0685-2.

7. Yildiz Y, Dursun A, Tokatli A, Coskun T, Sivri S. Partial hydatidiform mole in a phenylketonuria patient treated with sapropterin dihydrochloride. Gynecol Endocrinol. 2017;33(1):19-20. https://doi.org/10.1080/09513590. 2016.1247796

8. Grange DK, Hillman RE, Burton BK, Yano S, Vockley J, Fong C-T, et al. Sapropterin dihydrochloride use in pregnant women with phenylketonuria: an interim report of the PKU MOMS sub-registry. Mol Genet Metab. 2014;112(1):9-16. https://doi.org/10.1016/j.ymgme.2014.02.016.

9. Feillet F, Muntau AC, Debray F-G, Lotz-Havla AS, Puchwein-Schwepcke A, Fofou-Caillierez MB, et al. Use of sapropterin dihydrochloride in maternal phenylketonuria: a European experience of eight cases. J Inherit Metab Dis. 2014;37(5):753-62. https://doi.org/10.1007/s10545-014-9716-5.

10. Burton H, Sanderson S, Shortland G, Lee P. Needs assessment and review of services for people with inherited metabolic disease in the United Kingdom. J Inherit Metab Dis. 2006;29(5):667-76. https://doi.org/10.1007/ s10545-006-0374-0.

11. ten Hoedt AE, de Sonneville LMJ, Francois B, ter Horst NM, Janssen MCH, Rubio-Gozalbo ME, et al. High phenylalanine levels directly affect mood and sustained attention in adults with phenylketonuria: a randomised, double-blind, placebo-controlled, crossover trial. J Inherit Metab Dis. 2011;34(1):165-71. https://doi.org/10.1007/s10545-010-9253-9.

12 Burton BK, Leviton L, Vespa H, Coon H, Longo N, Lundy BD, et al. A diversified approach for PKU treatment: routine screening yields high incidence of psychiatric distress in phenylketonuria clinics. Mol Genet Metab. 2013;108(1):8-12. https://doi.org/10.1016/j.ymgme.2012.11.003.

13. Bilder DA, Burton BK, Coon H, Leviton L, Ashworth J, Lundy BD, et al. Psychiatric symptoms in adults with phenylketonuria. Mol Genet Metab. 2013;108(3):155-60. https://doi.org/10.1016/j.ymgme.2012.12.006.

14. Brumm VL, Azen C, Moats RA, Stern AM, Broomand C, Nelson MD, et al. Neuropsychological outcome of subjects participating in the PKU adult collaborative study: a preliminary review. J Inherit Metab Dis. 2004;27(5):549-66. https://doi.org/10.1023/b:boli.0000042985.02049.ff.

15 Ris MD, Weber AM, Hunt MM, Berry HK, Williams SE, Leslie N. Adult psychosocial outcome in early-treated phenylketonuria. J Inherit Metab Dis. 1997. https://doi.org/10.1023/a:1005389110739.

16 Pietz J, Fätkenheuer B, Burgard P, Armbruster M, Esser G, Schmidt H. Psychiatric disorders in adult patients with early-treated phenylketonuria. Pediatrics. 1997;99(3):345-50. https://doi.org/10.1542/peds.99.3.345.

17. Wolff OH, Smith I, Carson N, Graham PJ, Komrower GM, Turnbull AC, et al. Routine neonatal screening for phenylketonuria in The United Kingdom 1964-78: medical research council steering committee for the MRC/ DHSS Phenylketonuria Register. Br Med J. 1981;282(6277):1680-4.

18 Ashe K, Kelso W, Farrand S, Panetta J, Fazio T, De Jong G, et al. Psychiatric and cognitive aspects of phenylketonuria: the limitations of diet and promise of new treatments. Front Psychiatry. 2019;10:561. https://doi.org/ 10.3389/fpsyt.2019.00561.

19 Blonde L, Khunti K, Harris SB, Meizinger C, Skolnik NS. Interpretation and impact of real-world clinical data for the practicing clinician. Adv Ther. 2018;35(11):1763-74. https://doi.org/10.1007/s12325-018-0805-y.

20 Gluud LL. Bias in clinical intervention research. Am J Epidemiol. 2006;163(6):493-501. https://doi.org/10.1093/aje/kwj069.

21 Vardy ERLC, MacDonald A, Ford S, Hofman DL. Phenylketonuria, comorbidity, and ageing: a review. J Inherit Metab Dis. 2020;43(2):167-78. https://doi.org/10.1002/jimd.12186.

22. Burgess NM, Kelso W, Malpas CB, Winton-Brown T, Fazio T, Panetta J. The effect of improved dietary control on cognitive and psychiatric 
functioning in adults with phenylketonuria: the ReDAPT study. Orphanet J Rare Dis. 2021. https://doi.org/10.1186/s13023-020-01668-2.

23 Hogan WR, Wagner MM. Accuracy of data in computer-based patient records. J Am Med Inform Assoc. 1997;4(5):342-55. https://doi.org/10. 1136/jamia.1997.0040342.

24 Altman DG, Royston JP. The hidden effect of time. Stat Med. 1988;7(6):629-37. https://doi.org/10.1002/sim.4780070602.

25 Bilder DA, Kobori JA, Cohen-Pfeffer JL, Johnson EM, Jurecki ER, Grant ML. Neuropsychiatric comorbidities in adults with phenylketonuria: a retrospective cohort study. Mol Genet Metab. 2017;121(1):1-8. https://doi.org/ 10.1016/j.ymgme.2017.03.002.

26 Smith I, Knowles J. Behaviour in early treated phenylketonuria: a systematic review. Eur J Pediatr. 2000;159(Suppl 2):S89-93. https://doi.org/10. 1007/pl00014392.

\section{Publisher's Note}

Springer Nature remains neutral with regard to jurisdictional claims in published maps and institutional affiliations.

- fast, convenient online submission

- thorough peer review by experienced researchers in your field

- rapid publication on acceptance

- support for research data, including large and complex data types

- gold Open Access which fosters wider collaboration and increased citations

- maximum visibility for your research: over 100M website views per year

At BMC, research is always in progress.

Learn more biomedcentral.com/submissions 